\title{
UCRL-JRNL-225669
}

LAW RENCE LIVERMORE N A T IO N A L LABORATORY

Mutagenic Potency of

Food-Derived Heterocyclic Amines

J. S. Felton, M. G. Knize, R. W. Wu, M. E. Colvin,

F. T. Hatch, M. A. Malfatti

October 27, 2006

Mutation Research 
This document was prepared as an account of work sponsored by an agency of the United States Government. Neither the United States Government nor the University of California nor any of their employees, makes any warranty, express or implied, or assumes any legal liability or responsibility for the accuracy, completeness, or usefulness of any information, apparatus, product, or process disclosed, or represents that its use would not infringe privately owned rights. Reference herein to any specific commercial product, process, or service by trade name, trademark, manufacturer, or otherwise, does not necessarily constitute or imply its endorsement, recommendation, or favoring by the United States Government or the University of California. The views and opinions of authors expressed herein do not necessarily state or reflect those of the United States Government or the University of California, and shall not be used for advertising or product endorsement purposes. 
"Carrano Issue" - Mutation Research

\section{Mutagenic Potency of Food-Derived Heterocyclic Amines.}

James S. Felton ${ }^{1 *}$, Mark G. Knize ${ }^{1}$, Rebekah W. Wu ${ }^{1}$, Michael E. Colvin ${ }^{2}$, Frederick T. Hatch $^{3}$ and Michael A. Malfatti ${ }^{1}$

${ }^{1}$ Chemistry, Materials and Life Sciences Directorate, Lawrence Livermore National Laboratory, 7000 East Ave. Livermore, CA 94551, ${ }^{2}$ School of Natural Sciences, University of California, Merced, CA, and ${ }^{3} 27$ Pease Rd. Meridith NH 03253.

*To whom correspondence should be addressed

Phone: 925-422-5656

FAX: 925-422-2282

Email: felton1@1lnl.gov 


\begin{abstract}
The understanding of mutagenic potency has been primarily approached using "quantitative structure activity relationships" (QSAR). Often this method allows the prediction of mutagenic potency of the compound based on its structure. But it does not give the underlying reason why the mutagenic activities differ. We have taken a set of heterocyclic amine structures and used molecular dynamic calculations to dock these molecules into the active site of a computational model of the cytochrome P-450 1A1 enzyme. The calculated binding strength using Boltzman distribution constants was then compared to the QSAR value (HF/6-31G* optimized structures) and the Ames/Salmonella mutagenic potency. Further understanding will only come from knowing the complete set of mutagenic determinants. These include the nitrenium ion half-life, DNA adduct half-life, efficiency of repair of the adduct, and ultimately fixation of the mutation through cellular processes. For two isomers, $\mathrm{PhIP}$ and 3-Me-PhIP, we showed that for the 100-fold difference in the mutagenic potency a 5-fold difference can be accounted for by differences in the P450 oxidation. The other factor of 20 is not clearly understood but is downstream from the oxidation step. The application of QSAR (chemical characteristics) to biological principles related to mutagenesis is explored in this report.
\end{abstract}




\section{Introduction}

In identifying the compounds responsible for the mutagenic potency of cooked meats, a series of aromatic amines were synthesized at Lawrence Livermore National Laboratory, USA [1], in Japan [2] and in Sweden [3]. Early on, it was surprising to note that small changes in molecule structure had a large impact on mutagenic potency in the Ames/Salmonella test. This was especially apparent in the frameshift sensitive Salmonella strains TA1538 and TA98 [4,5]. More than 20 mutagenic aromatic amines have been identified from food and additional closely-related structural analogs and isomers have been synthesized to do structure-activity studies.

\section{Mutagenic Potency of Cooked-Food Mutagens}

There are two classes of aromatic amines related to cooked food mutagens: aminocarbolines, and amino-imidazoazaarenes (AIA). A group of 23 amino carbolines were evaluated for mutagenicity with a resulting range in mutagenic potency of over 200,000 fold. These studies found that potency depended on the presence and position of a pyridine-type nitrogen in one of the rings, the position of the amino group, and the methyl substitutions on ring carbon atoms [6]. The AIA class of mutagens comprises the most prevalent mutagenic compounds in well-done meat, and is therefore the most relevant for human exposures from foods. This class of mutagens is derived from creatine (a constituent of muscle), which contributes the amino group on the 2-carbon of the imidazole ring common to these AIAs. These AIAs have mutagenic potencies ranging 4.8 million-fold as seen in Figure 1. Three of the most commonly studied AIAs, PhIP, 
MeIQx, and IQ, are found in well-done meat. The structural parameters that determine their mutagenic potency were determined to be: the number of fused rings, the number of heteroatoms in the non-imidazole ring, N-methyl substitution on the imidazole ring, and methyl substitution on ring carbon atoms [6]. Figure 1 shows the more potent compounds have a heteroatom in the non-imidazole ring and have an N-methyl group. Although the chemical attributes of the mutagens can be defined, how this relates to the biology of mutagenesis is largely unanswered.

A set of eleven amino-trimethyimidazopyridine (TMIP) isomers, ranging 600-fold in mutagenic potency, were examined using computational methods. The principal determinants of higher mutagenic potency in these isomeric amines are: (1) a small dipole moment, (2) the combination of ring fusion and having the N3-methyl group, (3) a lower calculated energy of the pi electron system (4), a smaller energy gap between the highest and lowest unoccupied molecular orbitals of the amine (5), and a more stable nitrenium ion [7].

A study of the relationship between the mutagenic potency in the Ames test and the carcinogenic potency in rodents of 34 aromatic amines shows that there is a significant quantitative relationship $(\mathrm{R}=0.66 \mathrm{P}<0.001)$ between the two measurements after the results are translated into the appropriate quantitative terms [8]. Again, the chemical parameters of the number of rings in the compound and methyl substitutions at carbon atoms were important determinants in the carcinogenic potencies. These structureactivity studies were extended to the evaluation of electronic and hydropathic factors for 
80 amines, and the main determinant of mutagenicity was the extent of the pi electron system, with smaller contributions from dipole moment, the calculated stability of the nitrenium ion, and hydrophobicity [9].

\section{Mutagenicity of Two PhIP Isomers}

The importance of the position of the N-methyl group of PhIP is a good case study. The mutagenic potency of PhIP (methyl group at the 1-position) and its 3-Me-PhIP isomer differs by $\sim 100$-fold in the Ames/Salmonella assay (Figure 2A). None of the calculated chemical parameters mentioned above would explain the differences seen between these two isomers. We hypothesized that the slight differences in structure are important for interaction of the compound with the enzyme active sites for oxidation or further phase II enzymatic conjugation (see Figure 2B). The data in Figure 2C shows that the slower oxidation step (conversion of the PhIP isomers to their N-OH intermediate) from the 3Me-PhIP isomer accounts for approximately 5 -fold of the 100 -fold difference in potency for the mutagenicity. The remaining 20 -fold difference can be seen clearly by the mutagenic potency of the NOH derivates in the Ames/Salmonella test (Figure 2D). Further experimental investigation of the reasons for these differences in mutagenic potency will be difficult, but should be done. Differences in the stability of the reactive intermediates, and how they fit into the $\mathrm{N}$-acetyltransferase or Sulfotransferase active sites may impact further activation. Stability of the adduct, removal of the adduct (see discussion below), and specificity for base targets in the histidinol dehyrogenase gene, may all impact the mutagenic potency. 


\section{Enzyme Activity Can Affect Mutagenic Potential}

One informative study from our laboratory has shown that DNA binding of PhIP in human intestine is dependent primarily on both cytochrome P4501A2 oxidation and conjugation by phase II enzymes [10]. The premise of these studies was to relate phenotypic and genotypic differences for these activities in the patients with quantitative DNA binding measurement after PhIP exposure. In addition, the amount of UDPglucuronosyl transferase (UDPGT)-mediated $\mathrm{N}^{2}$ glucuronidation of NOH-PhIP was measured by analysis of the glucuronide conjugates in the urine of the patients. The study revealed that individuals with high levels of urinary NOH-PhIP-N ${ }^{2}$-glucuronide and a fast cytochrome P4501A2 phenotype were more protected against DNA adducts and presumably mutations, compared to individuals with low urinary NOH-PhIP-N ${ }^{2}$ glucuronide and a slow cytochrome P4501A2 phenotype. We proposed that the UDPGT glucuronidation at the $\mathrm{N}^{2}$ position detoxifies the NOH-PhIP intermediate making it unavailable for further activation and DNA damage [10].

\section{Computer Modeling}

Another way to understand the importance of small structural changes on mutagenic potential is to study the computer docking of metabolite intermediates into the enzyme active sites of Phase II activating enzymes. Recently, Lau et al. investigated the ability of various heterocyclic amine reactive intermediates to fit into the $\mathrm{N}$-acetyltransferase (NAT2) active-site [11]. Using a NAT2 homology model constructed from available crystal structures, docking studies and quantum mechanical calculations of hydroxylated heterocyclic amines revealed that the observed differences in mutagenic activity between 
NOH-PhIP and NOH-MeIQ are not related to their acetylation reaction with NAT2. These results suggest that other metabolic steps or steps in the DNA adduct formation and/or repair process may be involved in determining the mutagenic potential of these compounds.

\section{DNA Repair Affects Mutagenic Potency}

The ability to repair the initial adduct or damage is also important for determining a compound's mutagenic potency. For heterocyclic amines the 3' cutting step of nucleotide excision repair (XPG) appears to play an important role in repair of DNA damage. Figure 3 shows a number of $\mathrm{CHO}$ cell lines with mutant repair genes and their impact on cell killing. The XPG mutant has not only a large impact on survival, but also remarkably increased mutation frequency when exposed to NOH PhIP (data not shown). Experiments to understand the role of chemical structure on the incision efficiency at the 3' and 5' sites for these bulky adducts formed from heterocyclic amine exposure would greatly increase our understanding of the repair process for these types of chemicals. Clearly, whether the adduct is in the major groove or the minor groove of DNA will impact the efficiency of repair. What is not known is how small isomeric differences in the heterocyclic amines impact the structure of the DNA adduct and how that relates to mutagenic potency. Of course the polymerase will also play a role in the nucleotide excision repair process, but again, do these small changes in structure impact this step leading to the observed differences in mutagenic potency?

\section{QSAR Studies}


A number of QSAR studies of the AIA food mutagens have found that high mutagenic potency is predicted by low-energy lowest unoccupied molecular orbital (LUMO) $[12,13]$. Our results show a reasonably strong correlation between LUMO energy and observed mutagenic potential of several heterocyclic amine mutagens (Figure 4). A lower LUMO energy means a higher electron affinity. Using the reasoning that electron withdrawing groups should lead to lower LUMO energies, two novel, highly mutagenic heterocyclic amine analogs have been proposed. For each compound the optimized structure and LUMO energies using ab initio quantum chemical methods were calculated. Using the linear relationship between LUMO energy and log mutagenic potency it was possible to extrapolate and predict the potencies of these novel compounds.

\section{Conclusions}

Understanding the cancer risk of human exposure to heterocyclic amines is a challenging problem because the compounds vary widely in potency and quantities present in the diet. Adding to this complex problem is the knowledge that these compounds occur as mixtures of varying ratios in cooked meats. Still, the majority of human epidemiology studies suggest that exposure to heterocyclic amines may be involved in the development of tumors at multiple sites in humans, including breast, colon and stomach[14].

This discussion points out that there is a lack of understanding of the important biological pathways leading to mutation for compounds like heterocyclic amines. Clearly, the first step in metabolism is important, but for the PhIP isomers it only explains $20 \%$ of the

difference. Stability of the intermediates and adducts, efficiency of repair, conversion to 
more active leaving groups (possibly nitrenium ions) may all be important. Our understanding of how the chemical criteria, like dipole moment, a lower calculated energy of the pi electron system, and/or a smaller energy gap between the amine's

highest and lowest unoccupied molecular orbitals, impacts mutagenic potency is an important effort for future research.

\section{Acknowledgements}

This work was performed under the auspices of the U.S. Department of Energy by the University of California, Lawrence Livermore National Laboratory under contract No. W-7405-Eng-48, and supported by NCI Grant CA55861. 


\section{References}

[1] M.G. Knize and J.S. Felton The synthesis of the cooked-beef mutagen 2-amino-1methyl-6-phenylimidazo[4,5-b]pyridine and its 3-methyl isomer, Heterocycles 24 (1986) 1815-1819.

[2] M. Nagao, K. Wakabayashi, H. Kasai, S. Nishimura and T. Sugimura Effect of methyl substitution on mutagenicities of 2-amino-3-methylimidazo[4,5f]quinoline, isolated from broiled sardine, Carcinogenesis 2 (1981) 1147-1149.

[3] S. Grivas Efficient synthesis of mutagenic imidazo[4,5-f]quinoxalin-2-amines via readily accessible 2,1,3-benzoselenadiazoles, Acta Chem Scand B40 (1986) 404406.

[4] J.S. Felton and M.G. Knize Occurrence, identification, and bacterial mutagenicity of heterocyclic amines in cooked food, Mutat. Res. 259 (1991) 205218.

[5] J.S. Felton, L.F. Bjeldanes and F.T. Hatch Mutagens in cooked foods: Metabolism and genetic toxicity, in: M. Friedman (Ed.), Nutritional and Toxicological Aspects of Food Safety, Advances in Experimental Medicine and Biology, Plenum Press, NY, 1984, pp. 555-566.

[6] F.T. Hatch, M.G. Knize and J.S. Felton Quantitative structure-activity relationships of heterocyclic amine mutagens formed during the cooking of food, Environ. Molec. Mutagenesis 17 (1991) 4-19.

[7] M.G. Knize, F.T. Hatch, M.J. Tanga, E.Y. Lau and M.E. Colvin A QSAR for the mutagenic potencies of twelve 2-amino-trimethylimidazopyridine isomers: structural, quantum chemical, and hydropathic factors, Environ Mol Mutagen 47 (2006) 132-146.

[8] F.T. Hatch, M.G. Knize, D.H. Moore and J.S. Felton Quantitative correlation of mutagenic and carcinogenic potencies for heterocyclic amine from cooked foods and additional aromatic amines, Mutat. Res. 271 (1992) 269-287.

[9] F.T. Hatch, M.G. Knize and M.E. Colvin Extended quantitative structure-activity relationships for 80 aromatic and heterocyclic amines: structural, electronic, and hydropathic factors affecting mutagenic potency, Environ Mol Mutagen 38 (2001) 268-291.

[10] M.A. Malfatti, K.H. Dingley, S. Nowell-Kadlubar, E.A. Ubick, N. Mulakken, D. Nelson, N.P. Lang, J.S. Felton and K.W. Turteltaub The urinary metabolite profile of the dietary carcinogen 2-amino-1-methyl-6-phenylimidazo[4,5b]pyridine in predicitive of colon DNA adducts after a low-dose expsoure in humans, Cancer Research in press (2006).

[11] E.Y. Lau, J.S. Felton and F.C. Lightstone Insights into the O-acetylation reaction of hydroxylated heterocyclic amines by human arylamine $\mathrm{N}$-acetyltransferases: A computational study, Chemical Research in Toxicology 19 (2006) 1182-1190.

[12] F.T. Hatch, M.E. Colvin and E.T. Seidl Structural and quantum chemical factors affecting mutagenic potency of aminoimidazo-azaarenes, Environ Mol Mutagen 27 (1996) 314-330.

[13] Y.P. Zhang, G. Klopman and H.S. Rosenkranz Structural basis of the mutagenicity of heterocyclic amines formed during the cooking processes, Environ Mol Mutagen 21 (1993) 100-115. 
[14] M.G. Knize and J.S. Felton Formation and human risk of carcinogenic heterocyclic amines formed from natural precursors in meat, Nutrition Reviews 63 (2005) 158-165. 


\section{Figure Legend}

Figure 1. Chemical structure and common name of aminoimidazoazaarene mutagens ranging 4.8 million-fold in mutagenic potency. Revertant colonies in Salmonella strain TA98 per nanomole are given.

Figure 2. Mutagenic difference between two PhIP isomers. A: Mutagenic potency of PhIP (methyl group at the 1-posion) and 3-Me-PhIP. B: Metabolic activation scheme for PhIP. C: Formation of NOH-PhIP from PhIP and 3-me-PhIP isomers exposed to rat liver microsomes. D: Direct acting mutagenicity of NOH-PhIP and 3-me-NOH-PhIP in the Ames/Salmonella assay.

Figure 3. Cytotoxicity of NOH-PhIP in various CHO cell lines with mutant DNA repair genes.

Figure 4. Linear relationship between LUMO energy and log mutagenic potency from QSAR involving 15 AIA mutagens. Each point represents one AIA compound. From this data extrapolation of the mutagenic potency of two proposed novel highly mutagenic heterocyclic amine analogs, with lower LUMO energies than any observed AIA, is shown. A lower LUMO energy means higher electron affinity. 


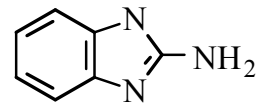

Amino benzimidazole, 0.13

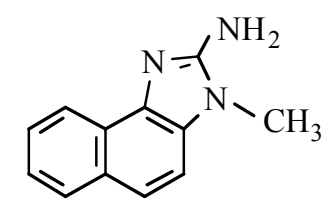

Amino 3-Me-naphthalene, 3.89

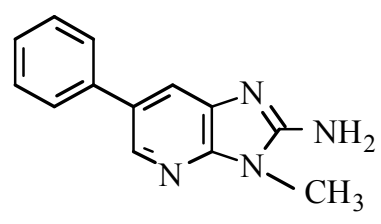

3-Me PhIP, 4.47

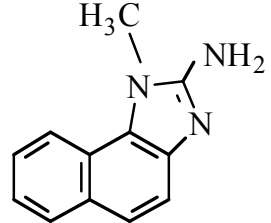

Amino 1-Me-naphthalene, 195

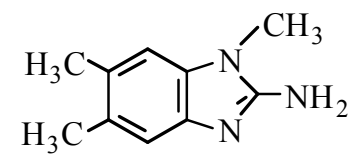

Amino Trimethylbenzimidazole, 347

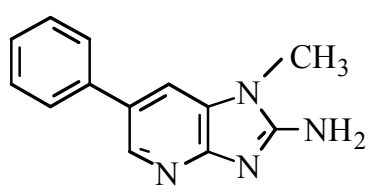

PhIP, 427<smiles>Cc1cnc2ccc3c(nc(N)n3C)c2n1</smiles>

MeIQx, 20,000<smiles>Cn1c(N)nc2c3cccnc3ccc21</smiles>

IQ, 50,000

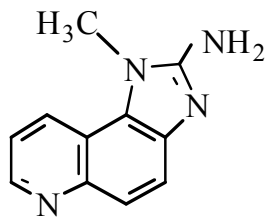

1-Me-IQ, 630,000

Figure 1 
A<smiles>Cn1c(N)nc2ncc(-c3ccccc3)cc21</smiles>

PhIP

Mutagenic Potency $1800 \mathrm{rev} / \mathrm{\mu g}$<smiles>Cn1c(N)nc2cc(-c3ccccc3)cnc21</smiles>

3-Me-PhIP

Mutagenic Potency

$20 \mathrm{rev} / \mu \mathrm{g}$
B

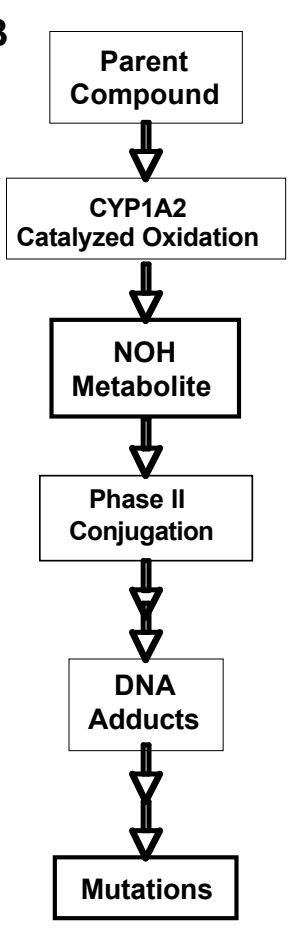

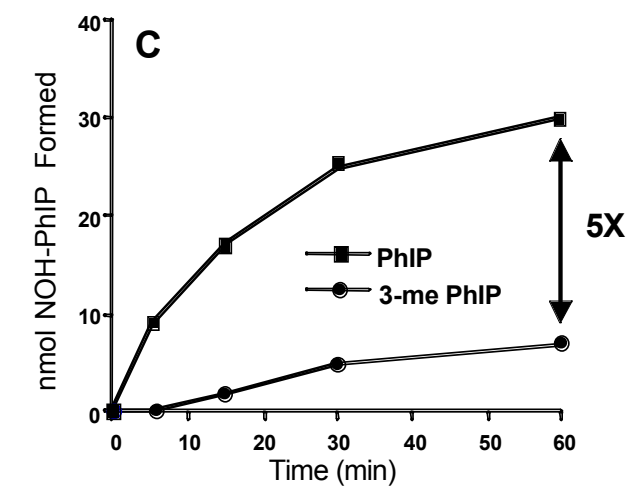

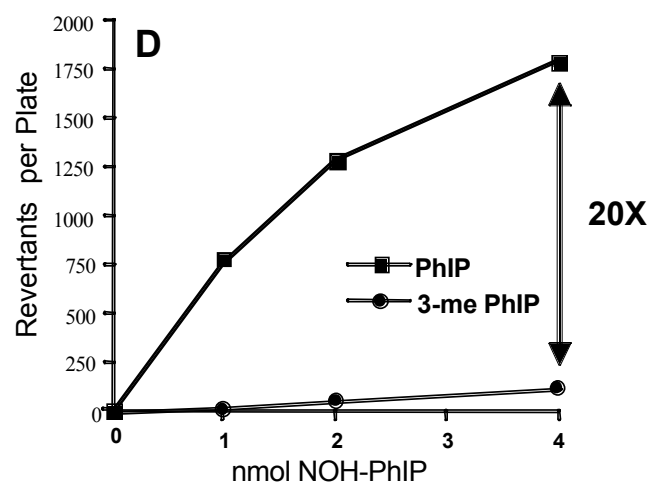

Figure 2

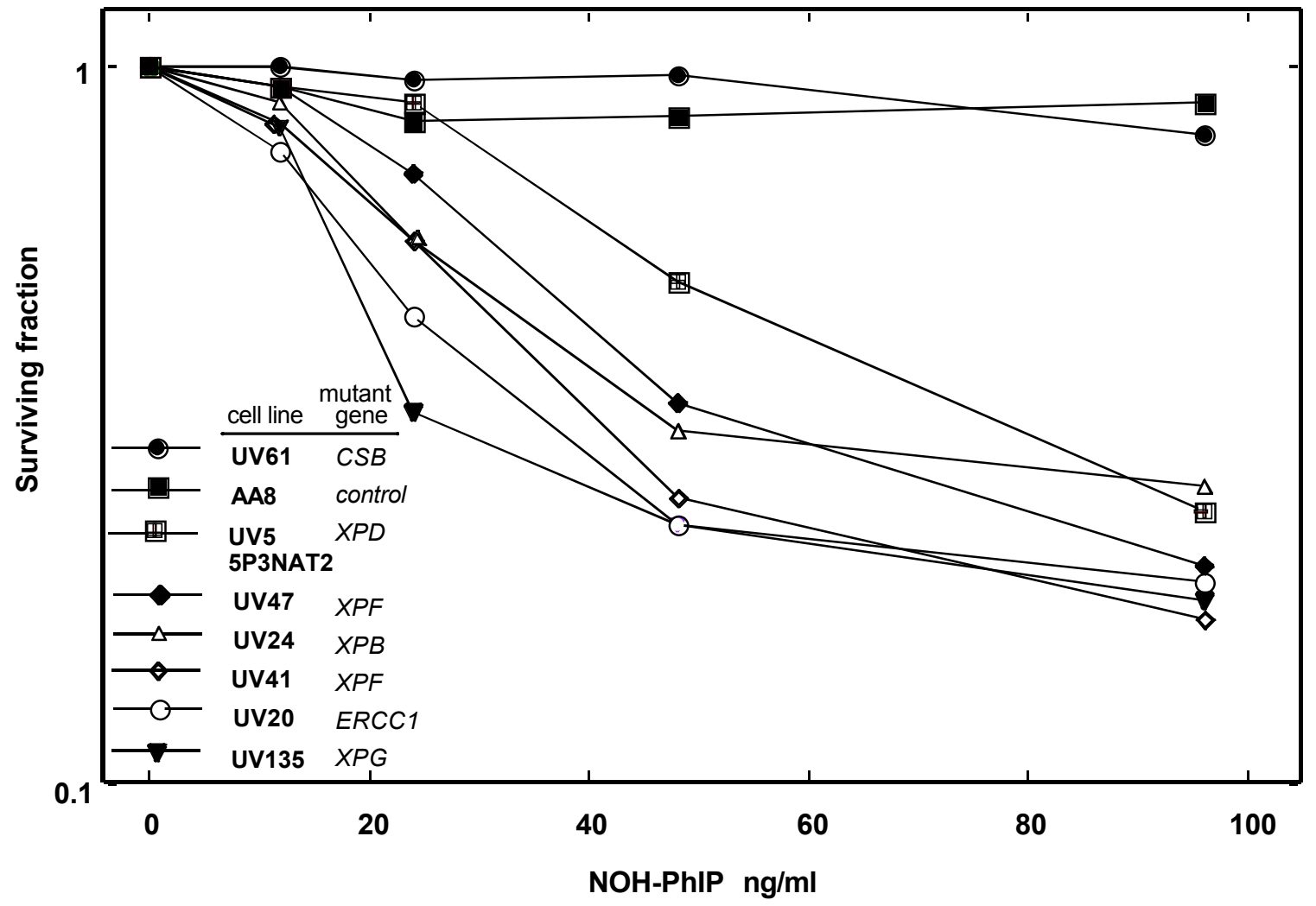

Figure 3 


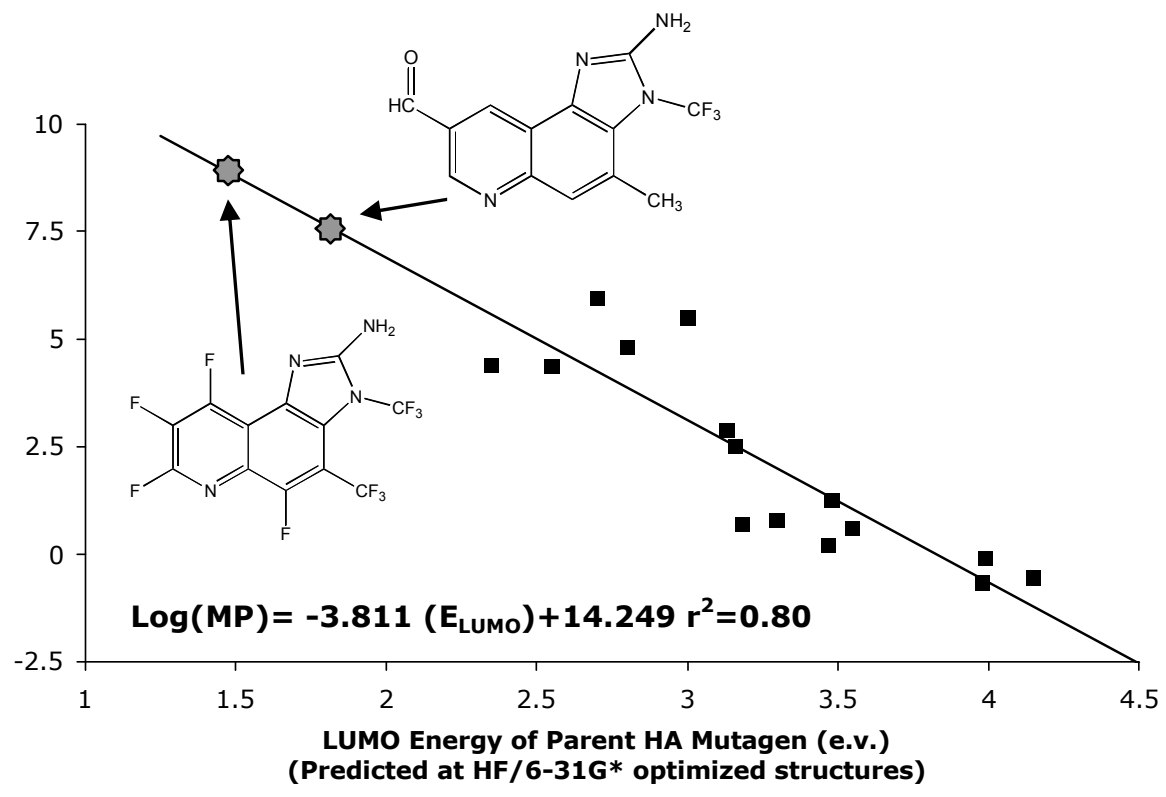

Figure 4 
